ИЗВЕСТИЯ АКАДЕМИИ НАУК ЭСТОНСКОП ССР. ТОМ 27 ХИМИЯ. 1978, № 4

\title{
РАСЧЕТ РАВНОВЕСИЯ ЖИДКОСТЬ-ПАР В МНОГОКОМПОНЕНТНЫХ СИСТЕМАХ
}

Большой объем необходимой экспериментальной работы и трудности исследования равновесия между жидкостью и паром в многокомпонентных системах (МКС), обусловленные часто сложностью определения состава пара, сделали актуальной разработку методов расчета по данным о бинарных системах. К числу недостатков наиболее часто используемых методов расчета, основанных на применении уравнения ДюгемаМаргулеса и эмпирических и полуэмпирических зависимостей свободной энергии Гиббса от состава раствора, следует отнести громоздкость вычислений. Возникает также вопрос о критерии выбора формы названной зависимости, которая позволила бы наиболее точно воспроизвести экспериментальные данные ['].

Изложенный в настоящем сообщении метод расчета равновесия жидкость-пар применим к МКС, зависимость относительной летучести $\alpha_{i k}=\frac{y_{i} / y_{k}}{x_{i} / x_{k}}$ от состава раствора $\left(x_{i}\right)$ бинарных составляющих которых близка к линейной.

Зависимость логарифма относительной летучести двух компонентов такой МКС от переменных состава раствора, молярной доли компонента $i$ в бинарном растворителе $i k x_{i(i k)} \equiv \frac{x_{i}}{x_{i}+x_{k}} \quad$ и молярных долей остальных компонентов можно представить в дифференциальной форме:

$$
d \lg \alpha_{i k}=\left(\frac{\partial \lg \alpha_{i k}}{\partial x_{i(i k)}}\right)_{P(T), x_{1}, \ldots, x_{t-1}, x_{t+1}, \ldots, x_{k-1}, x_{k+1}, \ldots, x_{n}} d x_{i(i k)}+
$$

$$
+\sum_{\substack{j=1 \\ j \neq i \neq k}}^{n}\left(-\frac{\partial \lg \alpha_{i k}}{\partial x_{j}}\right)_{P(T), x_{l}(i k), x_{1}, \ldots, x_{l-1}, x_{l+1}, \ldots, x_{k-1}, x_{k+1}, \ldots, x_{l-1}, x_{j+1}, \ldots, x_{n}} d x_{j} .
$$

Предположив, что линейная зависимость относительной летучести от переменных состава раствора сохраняется и в МКС, проинтегрируем выражение (1) вдоль секущей $x_{i(i k)}=$ const в пределах от бинарного $\left(x_{i}^{i k}=x_{i(i k)}\right)$ до $n$-компонентного раствора

$$
\lg \alpha_{i k}^{1} \ldots n=\lg \alpha_{i k}^{i k}+\sum_{\substack{j=1 \\ j \neq i \neq k}}^{n}\left(\frac{\partial \lg \alpha_{i k}}{\partial x_{j}}\right)_{P(T), x_{t}^{\prime k}} x_{j} .
$$


Приписав в уравнении (2) индекс $i$ одному из компонентов и циклически заменяя индексы остальных, получим необходимую для расчета состава $n$-компонентного пара систему $n-1$ уравнений (2). Такой подход дает $n$ вариантов и $n$ результатов расчета.

Предположением о линейной зависимости $\lg \alpha_{i k}$ от $x_{j}$ можно воспользоваться также для расчета значений частных производных

$$
\frac{\partial \lg \alpha_{i k}}{\partial x_{j}} \approx \lg \alpha_{i k}^{(j)}-\lg \alpha_{i k}^{i k}=\lg \alpha_{i j}^{(j)}-\lg \alpha_{k j}^{(j)}-\lg \alpha_{i k}^{i k} .
$$

Предельные значения относительных летучестей $\left(\alpha^{(j)}\right)$ и значение относительной летучести в исходной точке $\left(\alpha_{i k}^{i k}\right)$ можно определить из данных по бинарным системам, составляющим тройную $i k j$, представленных графически или аналитически.

Для расчета температуры кипения многокомпонентного раствора можно воспользоваться уравнением

$$
t_{1} \ldots n=t_{i k}+\sum_{\substack{j=1 \\ j \neq i \neq k}}^{n}\left(\frac{\partial t}{\partial y_{j}}\right)_{P, y_{t}^{t_{k}}} y_{j}
$$

которое получено, исходя из предположения, что в МКС сохраняется линейная зависимость температуры сосуществования жидкости и пара от переменных состава пара (yі(ik) и молярных долей остальных компонентов), которая характерна для многих бинарных систем.

Значения $t_{i k}$ (для $\left.y_{i}^{i k}=y_{i(i k)}\right)$ и $\partial t / \partial y_{j}$ рассчитаны также на основе этого предположения из данных о зависимости температуры конденсации от состава пара в бинарных системах, составляющих тройную $i k j$ : при наличии бинарных азеотропов

$$
\begin{aligned}
& t_{i k}=t_{i}+\frac{t_{i k}^{\mathrm{az.}}-t_{i}}{\left(x_{k}^{i k}\right)^{\text {a3. }}}\left(1-y_{i(i k)}\right) \\
& \frac{\partial t}{\partial y_{j}}=t_{i}+\frac{t_{i j}^{\mathrm{a3.}}-t_{i}}{\left(x_{j}^{i j}\right)^{\text {a3. }}}-t_{i k} \\
& t_{i k}=t_{k}+\frac{t_{i k}^{\text {a3. }}-t_{k}}{\left(x_{i}^{i k}\right)^{\text {a3. }}} y_{i(i k)} \\
& \frac{\partial t}{\partial y_{j}}=t_{k}+\frac{t_{k j}^{\text {a3. }}-t_{k}}{\left(x_{j}^{k j}\right)^{\text {aз. }}}-t_{i k}
\end{aligned}
$$

при отсутствии бинарных азеотропов

$$
\begin{aligned}
& t_{i k}=t_{k}+\left(t_{i}-t_{k}\right) y_{i(i k)}, \\
& \frac{\partial t}{\partial y_{j}}=t_{j}-t_{k} .
\end{aligned}
$$


Уравнения для расчета давления пара МКС получаются путем замены $t$ на $P$ (или на $\lg P$ ) в выражениях (4)-(6).

Сравнение результатов эксперимента и расчета данных о равновесии жидкость-пар в многокомпонентных системах

\begin{tabular}{|c|c|c|c|c|c|c|c|c|c|}
\hline \multirow{3}{*}{$\begin{array}{c}\text { №.№ } \\
\Pi / \Pi\end{array}$} & \multirow{3}{*}{ Система } & \multicolumn{4}{|c|}{ Метод Вильсона } & \multicolumn{4}{|c|}{ ІІредлагаемый метод } \\
\hline & & \multicolumn{2}{|c|}{$\Delta y_{i}$} & \multicolumn{2}{|c|}{$\Delta t$} & \multicolumn{2}{|c|}{$\Delta y_{i}$} & \multicolumn{2}{|c|}{$\Delta t$} \\
\hline & & сред. & макс. & сред. & макс. & сред. & макс. & сред. & макс. \\
\hline
\end{tabular}

1. Гексан (1) $\left.{ }^{[2}\right] \quad 0,007 \quad 0,013$ метилциклопентан (2) бензол (3) циклогексан (4) толуол (5)

2. Ацетон (1) [3] метилацетат (2) хлороформ (3) метанол (4) бензол (5)

3. Метилацетат
(1) [3] хлороформ (2) метанол (3) бензол (4)

4. Ацетон (1) $\left[{ }^{4}\right]$ 2,3-диметилбутан (2) хлороформ (3)

5. Ацетон (1) [5] метанол (2) изопропанол (3)

6. Бутанон (1) [7] бензол (2) изопропанол (3)

7. Муравьиная кислота (1) [ $\left.{ }^{8}\right]$ уксусная кислота (2) пропионовая кислота (3)

8. Вода (1) [ $\left.{ }^{9}\right]$ муравьиная кислота (2) уксусная кислота (3)

9. Вода (1) [11] муравьнная кислота (2) уксусная кислота (3) пропионовая кислота (4)

$\begin{array}{ll}0,006 & 0,028 \\ 0,008 & 0,012 \\ 0,003 & 0,007 \\ 0,005 & 0,016 \\ 0,005 & 0,018 \\ & \\ 0,005 & 0,012 \\ 0,004 & 0,017 \\ 0,012 & 0,033 \\ 0,005 & 0,030\end{array}$

$\begin{array}{ll}0,004 & 0,01 \\ 0,005 & 0,01 \\ 0,008 & 0,02 \\ 0,004 & 0,01 \\ 0,011 & 0,02 \\ 0,010 & 0,02 \\ 0,005 & 0,02 \\ 0,006 & \\ 0,005 & \\ 0,005 & \end{array}$

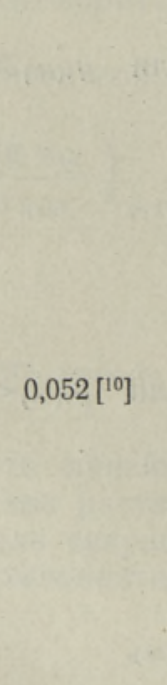

\begin{tabular}{|c|c|c|c|c|c|}
\hline & & 0,003 & 0,006 & & \\
\hline 0,52 & 0,82 & $\begin{array}{l}0,007 \\
0,003 \\
0,004 \\
0,006\end{array}$ & $\begin{array}{l}0,025 \\
0,007 \\
0,008 \\
0,010\end{array}$ & 0,38 & 1,89 \\
\hline & & 0,007 & 0,020 & & \\
\hline 0,39 & 0,82 & $\begin{array}{l}0,010 \\
0,007 \\
0,018 \\
0,006\end{array}$ & $\begin{array}{l}0,035 \\
0,045 \\
0,033 \\
0,033\end{array}$ & 0,35 & 1,66 \\
\hline
\end{tabular}

$\begin{array}{llllll}0,25 & 0,71 & 0,008 & 0,018 & & \\ & 0,007 & 0,025 & 0,56 & 1,25 \\ & 0,009 & 0,028 & & \\ & 0,005 & 0,017 & & \\ & & 0,008 & 0,017 & & \end{array}$

$\begin{array}{llllll}0,63 & 1,0 & 0,006 & 0,023 & 1,47 & 3,4\end{array}$

$\begin{array}{rllll}\left.5,3 *{ }^{6}\right] & 0,005 & 0,013 & & \\ 0,005 & 0,014 & 8,4^{*} & \\ & 0,004 & 0,010 & & \\ 0,011 & 0,022 & & \\ 0,008 & 0,027 & 3,9 * & 11,3 *\end{array}$

$0,007 \quad 0,020$

$0,008 \quad 0,037$

$\begin{array}{llll}0,003 & 0,029 & 2,0 & 3,7\end{array}$

$0,008 \quad 0,022$

$0,009 \quad 0,027$

$\begin{array}{llll}0,009 & 0,039 & 0,73 & 1,5\end{array}$

$0,008 \quad 0,024$

$0,014 \quad 0,042$

$0,013 \quad 0,059$

$0,007 \quad 0,034$

$0,018 \quad 0,044$

* Давление пара. 
В таблице результаты расчета по предлагаемому методу в форме среднеарифметических и максимальных абсолютных расхождений с экспериментальными данными сопоставлены для ряда систем с результатами расчета по методу Вильсона. Для большинства систем последние, наряду с экспериментальными данными, заимствованы из работ, ссылки на которые указаны в таблице. Для системы № 5 среднеарифметические абсолютные расхождения эксперимента и расчета по уравнению Вильсона взяты из работы [6]. Для системы № 8, в основу расчета которой по предлагаемому методу положены данные о бинарных составляющих из справочника [12] (таблица, №№ 245, 287, 672), усредненные по всем компонентам абсолютные расхождения между экспериментом (таблица, № $\left.1895\left[{ }^{12}\right]\right)$ и расчетом по методу Вильсона заимствованы из работы $\left[{ }^{10}\right]$. Слишком большое расхождение $(5,2$ мол. \%) вызывает подозрение, что здесь опечатка, допущенная в этой работе. Для систем №№ 7 и 9 мы не нашли в литературе результатов расчета по уравнению Вильсона, а для системы № 6, для которой здесь использованы изотермические экспериментальные данные [7], в статье с результатฺами расчета по уравнению Вильсона сопоставлены изобарические данные из другого источника $\left[{ }^{13}\right]$.

Из данных, приведенных в таблице, видно, что предлагаемый метод уступает в точности расчету по методу Вильсона только в случае МКС, в которых зависимость $\lg \alpha_{i k}=f\left(x_{i}^{i k}\right)$ некоторых бинарных составляющих значительно отклоняется от линейной (в системах №№ 2, 3 бинарная составляющая $2-3)$.

Метод применим как к изобарическим, так и к изотермическим условиям. Техника расчета проста. Текст программы на языке Фортран для расчета равновесия МКС на ЭВМ приведен ниже (см. приложение).

Принятые обозначения:

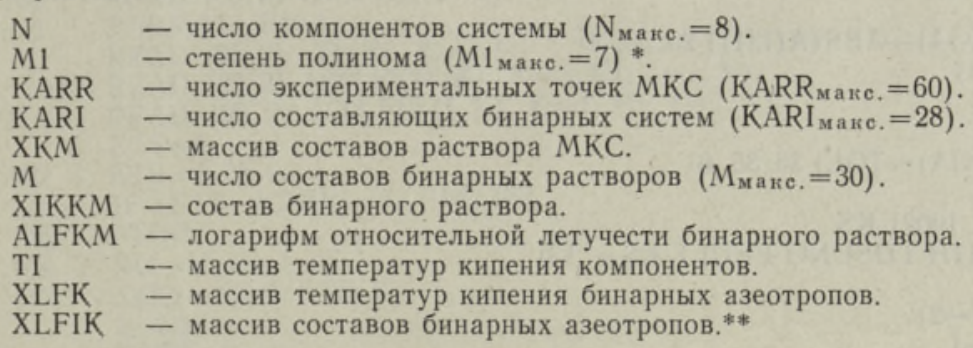
единице.

* При расчетах в настоящей работе степень полинома была принята равной

** Расчет температур кипения (давления пара) на ЭВМ осуществляется параллельно по формулам (5) и (6). Путем сопоставления с экспериментальными данными нетрудно убедиться, что при наличии бинарных азеотропов расчет (5) более точен.

Приложение

SUBROUTINE POL (N, $M$, iTT)

DIMENSION A(64), XAS (16)

COMMON/BL1/X (30), Y (30), B (8)

$93 \quad \mathrm{XAS}(\mathrm{J})=0.0$

$\mathrm{J}=1$

$\mathrm{I}=1$

$94 \quad \mathrm{XAS}(\mathrm{J})=\mathrm{XAS}(\mathrm{J})+\mathrm{X}(\mathrm{I}) * * \mathrm{~J}$

$\mathrm{I}=\mathrm{I}+1$

IF (I-M) 94, 94, 95

$\mathrm{J}=\mathrm{J}+1$

IF $\left(\mathrm{J}-2^{*}(\mathrm{~N}-1)\right) 93,93,96$ 


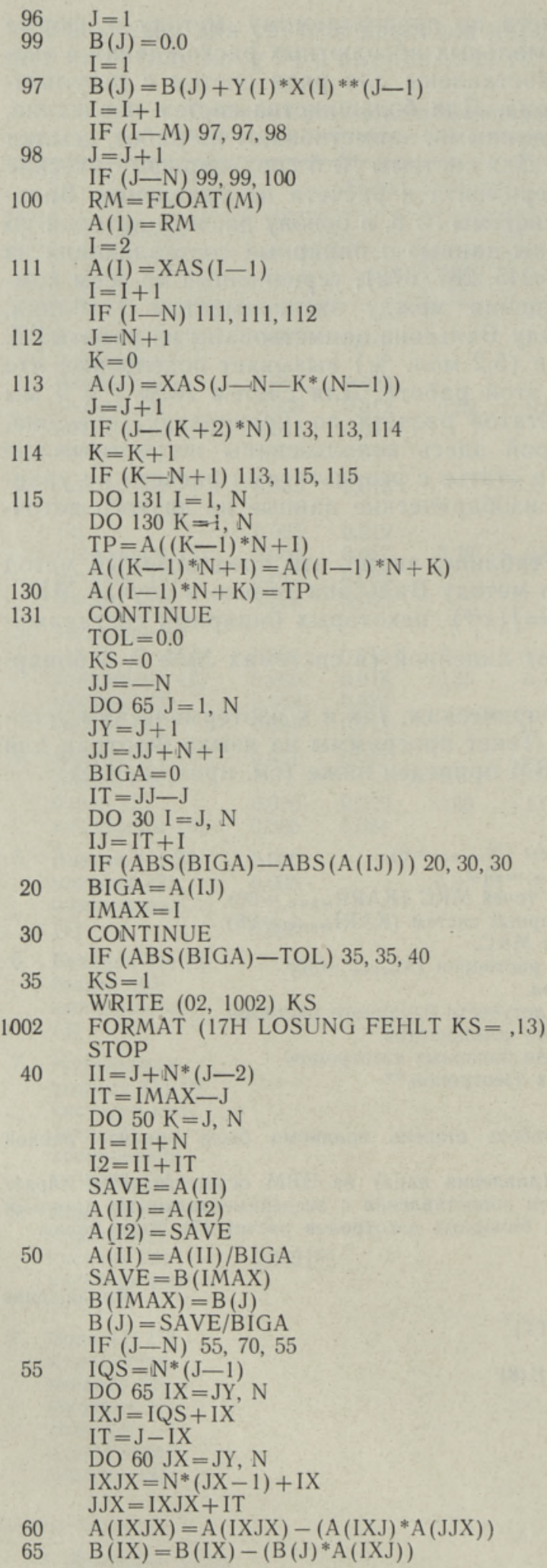


70

$\mathrm{NY}=\mathrm{N}-1$

$\mathrm{IT}=\mathrm{N} * \mathrm{~N}$

DO $80 \mathrm{~J}=1, \mathrm{NY}$

$\mathrm{IA}=\mathrm{IT}-\mathrm{J}$

$\mathrm{IB}=\mathrm{N}-\mathrm{J}$

$\mathrm{IC}=\mathrm{N}$

DO $80 \mathrm{~K}=1$, J

$\mathrm{B}(\mathrm{IB})=\mathrm{B}(\mathrm{IB})-\mathrm{A}(\mathrm{IA}) * \mathrm{~B}(\mathrm{IC})$

$80 \quad \mathrm{IC}=\mathrm{IC}-1$

41 DO $119 \mathrm{~J}=1, M$

$\mathrm{XAR}=\mathrm{B}(\mathrm{N})$

DO $117 \mathrm{I}=2, \mathrm{~N}$

$117 \quad \mathrm{XAR}=\mathrm{XAR} * \mathrm{X}(\mathrm{J})+\mathrm{B}(\mathrm{N}-\mathrm{I}+1)$

$\mathrm{V} 1=\mathrm{Y}(\mathrm{J})-\mathrm{XAR}$

IF (ITT) $119,119,118$

118 WRITE $(10,83) \mathrm{X}(\mathrm{J}), \mathrm{Y}(\mathrm{J}), \mathrm{XAR}, \mathrm{VI}$

119 CONTINUE

83 FORMAT (4F12.6)

RETURN

END

MAIN

DIMENSION XIKKM(28,30), $\operatorname{ALFKM}(28,30), \operatorname{XKM}(60,8), \operatorname{MKM}(28)$

DIMENSION YI (8)

DIMENSION ALFK $(7,8), \operatorname{ALFIK}(7,8)$

DIMENSION X $(8), \operatorname{TI}(8), \operatorname{XLFK}(7,8), \operatorname{XLFIK}(7,8)$

COMMON/BL1/XIK (30), ALFA(30), B(8)

$\mathrm{ITT}=2$

READ $(05,1) \mathrm{N}, \mathrm{M1}$, KARR, KARI

FORMAT (I 3)

$M 1=M 1+1$

$\operatorname{READ}(05,2) \quad((\mathrm{XKM}(\mathrm{I}, \mathrm{K}), \mathrm{K}=1, \mathrm{~N}), \mathrm{I}=1, \mathrm{KARR})$

DO $102 \mathrm{I}=1$, KARI

READ $(05,1) M$

$\operatorname{MKM}(\mathrm{I})=\mathrm{M}$

READ $(05,2)(X I K K M(I, K), K=1, M)$

102 READ $(05,2)(\operatorname{ALFKM}(\mathrm{I}, \mathrm{K}), \mathrm{K}=1, \mathrm{M})$

2 FORMAT (F10.4)

READ $(05,2) \quad(\mathrm{TI}(\mathrm{K}), \mathrm{K}=1, \mathrm{~N})$

$\operatorname{READ}(05,2) \quad((\operatorname{XLFK}(I, K), K=1, N), I=1, N)$

$\operatorname{READ}(05,2) \quad((\mathrm{XLFIK}(\mathrm{I}, \mathrm{K}), \mathrm{K}=1, \mathrm{~N}), \mathrm{I}=1, \mathrm{~N})$

107

$\mathrm{KTS}=0$

IF (KTS - KARR) 104, 104, 105

105 STOP

104 DO $117 \mathrm{~K}=1, \mathrm{~N}$

$117 \quad \mathrm{X}(\mathrm{K})=\mathrm{XKM}(\mathrm{KTS}, \mathrm{K})$

$\mathrm{IL}=0$

$\mathrm{KATA}=0$

$\mathrm{IL}=\mathrm{IL}+1$

IF $(\mathrm{IL}-\mathrm{N}) 3,8,8$

$3 \quad \mathrm{KL}=\mathrm{IL}+1$

$6 \mathrm{KATA}=\mathrm{KATA}+1$

$M=M K M($ KATA $)$

DO $101^{\circ} \mathrm{K}=1, M$

$\mathrm{XIK}(\mathrm{K})=\mathrm{XIKKM}(\mathrm{KATA}, \mathrm{K})$

$101 \quad \operatorname{ALFA}(\mathrm{K})=\mathrm{ALFKM}(\mathrm{KATA}, \mathrm{K})$

CALL POL ( $M 1, M$, ITT)

IF (ITT) $87,87,88$

88 WRITE $(10,30)$ IL, KL

30 FORMAT $(3 \mathrm{H} \mathrm{I}=, 12,3 \mathrm{H} \mathrm{K}=, 12)$

DO $4 \mathrm{~K}=1, \mathrm{M} 1$

WRITE $(10,5) \mathrm{K}, \mathrm{B}(\mathrm{K})$

5 FORMAT $(2 \mathrm{H} \mathrm{B}, 12,1 \mathrm{H}=, \mathrm{F} 12.6)$

WRITE $(10,41)$

41 FORMAT (1HO)

$87 \quad \mathrm{XKI}=\mathrm{X}(\mathrm{IL}) /(\mathrm{X}(\mathrm{IL})+\mathrm{X}(\mathrm{KL}))$

$\mathrm{PV}=\mathrm{B}(\mathrm{Ml})$

3 ENSV TA Toimetised. K 41978 


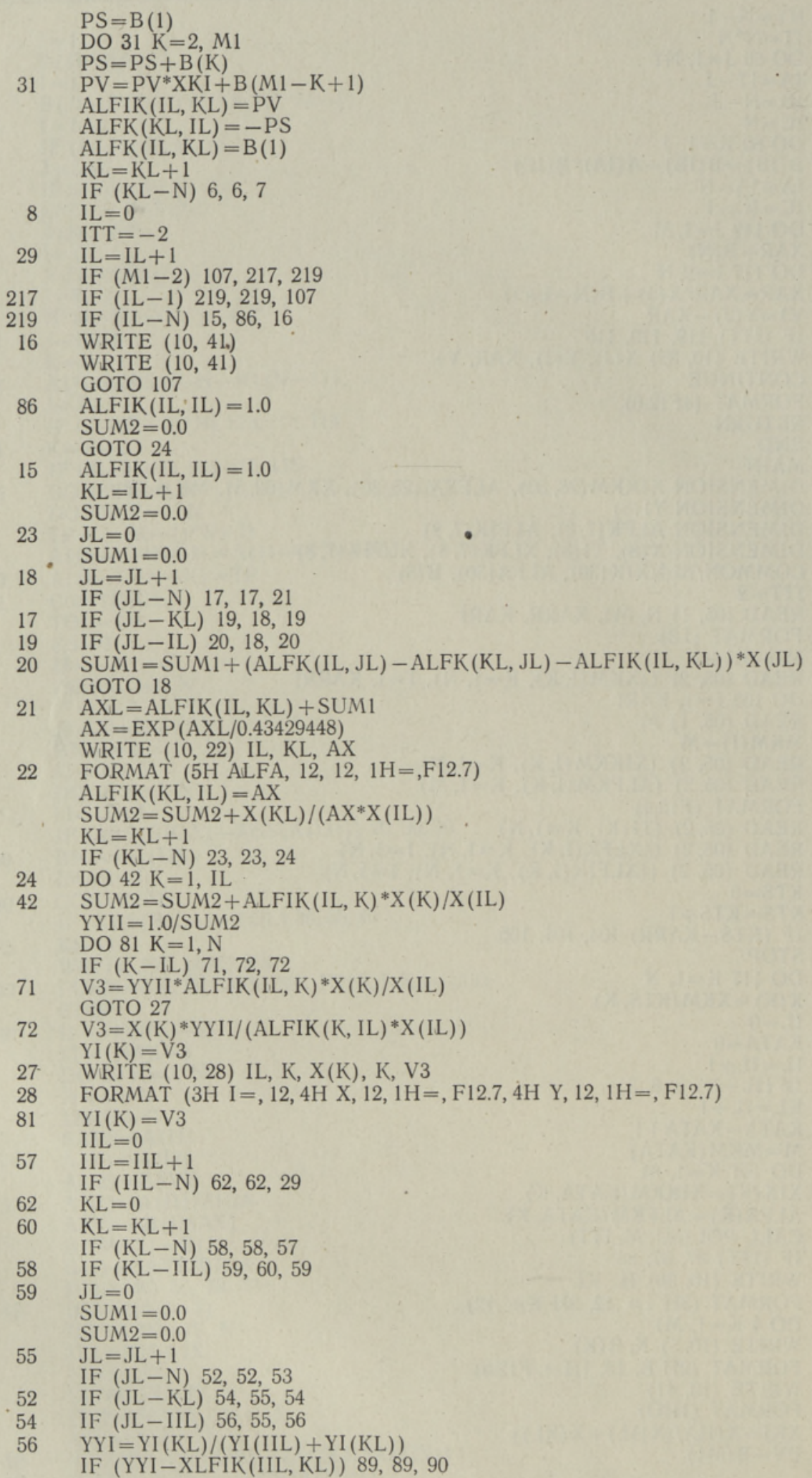


$\mathrm{V} 4=(\mathrm{XLFK}(\mathrm{IIL}, \mathrm{KL})-\mathrm{TI}(\mathrm{KL})) /(1.0-\mathrm{XLFIK}(\mathrm{IIL}, \mathrm{KL}))$

$\mathrm{TIK}=\mathrm{TI}(\mathrm{KL})+\mathrm{V} 4^{*}(1.0-\mathrm{YYI})$

$\mathrm{V} 3=(\mathrm{XLFK}(\mathrm{KL}, \mathrm{JL})-\mathrm{TI}(\mathrm{KL})) / \mathrm{XLFIK}(\mathrm{KL}, \mathrm{JL})$

$$
\mathrm{VV} 3=\mathrm{TI}(\mathrm{KL})
$$

$89 \quad \mathrm{~V} 4=(\mathrm{XLFK}(\mathrm{IIL}, \mathrm{KL})-\mathrm{TI}(\mathrm{IIL})) / \mathrm{XLFIK}(\mathrm{IIL}, \mathrm{KL})$

$$
\mathrm{V} 4=\mathrm{V} 4 * \mathrm{YYI}
$$

$\mathrm{TIK}=\mathrm{TI}(\mathrm{IIL})+\mathrm{V} 4$

$\mathrm{V} 3=(\mathrm{XLFK}(\mathrm{IIL}, \mathrm{JL})-\mathrm{TI}(\mathrm{IIL})) / \mathrm{XLFIK}(\mathrm{IIL}, \mathrm{JL})$

$\mathrm{VV} 3=\mathrm{TI}(\mathrm{IIL})$

$91 \mathrm{SUM} 1=\mathrm{SUM} 1+(\mathrm{VV} 3+\mathrm{V} 3-\mathrm{TIK}) * \mathrm{YI}(\mathrm{JL})$

SUM2 $=$ SUM2 $+($ TI $(J L)-$ TIK $) *$ YI $(J L)$

GOTO 55

$53 \quad \mathrm{TX}=\mathrm{TIK}+\mathrm{SUM} 1$

$\mathrm{TX} 1=\mathrm{TIK}+\mathrm{SUM} 2$

WRITE $(10,61)$ IIL, KL, TX, TX1

61 FORMAT (2H T, 12, 12, $1 \mathrm{H}=, 2 \mathrm{~F} 12.7)$

GOTO 60

END

FINISH

\section{ЛИТЕРА Т У Р А}

1. Ки р с с Х. Х., М а ри ни чев А. Н., Э йзен О. Г. Выбор метода обработки экспериментальных данных по равновесию жидкость-пар. - Ж. физ. химии, 1978, т. 52 , вып. 7 , с. $1817-1818$.

2. Weatherford, R. W., van Winkle, M. Vapor-liquid equilibria of the quinary system hexane, methylcyclopentane, cyclohexane, benzene and toluene. - J. Chem. Eng. Data, 1970, v. 15, N 3, p. 386-390.

3. Hudson, J. H., van Winkle, M. Multicomponent vapor-liquid equilibria in systems of mixed positive and negative deviations. - J. Chem. Eng. Data, 1969 , v. $14, \mathrm{~N} 3$, p. $310-316$.

4. Ga r ret, G. R., van Winkle, M. Vapor-liquid equilibria of the ternary system acetone-chloroform-2,3-dimethylbutane. - J. Chem. Eng. Data, 1967, v. $14, \mathrm{~N} 3$, p. $302-306$.

5. Freshw at er, D. C., Pike, K. A. Vapor-liquid equilibrium data for systems of acetone-methanol-isopropanol. - J. Chem. Eng. Data, 1967, v. 12, N 2, p. $179-183$.

6. Н а гат а M. Расчет данных по равновесию жидкость-пар в бинарах и тройных смесях с помощью уравнения Вильсона. - Kагаку когаку, 1969, т. 33, № 3, c. 263-267. [Перевод ВИНИТИ].

7. Ж аров В. Т., Малегин а Н. Д., Морачевский А. Г. Равновесие жидкость-пар в тройной системе метилэтилкетон-бензол-изопропиловый спирт. - Ж. прикл. химии, 1965, т. 38, № 9, с. 2132-2134.

8. Кушнер Т. М., Кришталь Е. Л., Т ациевская Г. И., Сера фимов Л. А. Исследование и расчет фазового равновесия в системе муравьиная кислотауксусная кислота-пропионовая кислота при атмосферном давлении. - Ж. физ. химии, 1967 , т. 41 , № 9, с. $2162-2167$.

9. Аристович В. Ю., Лутугина Н. В., Маленко Ю. И., Морачевский А. Г. Исследование равновесия жидкость-пар и процессов ректификации в тройной системе вода-муравьиная кислота-уксусная кислота. - Ж. прикл. химии, 1960 , т. 33 , № 12 , с. $2693-2698$.

10. Рудаковская Т. С., Николаев Е. С., Мозжухин А. С., Серафи м о в Л. А. Расчет парожидкостного равновесия с использованием аппроксимирующих зависимостей для параметров уравнения Вильсона. - Теор. основы хим. технол., 1977 , т. 11 , № 3, с. $454-457$.

11. Кушнер Т. М., Л ебедева Т. Н., Т ациевская Г. И., Сера фимов Л. А. Равновесие жидкость-пар в системе вода-муравьнная кислота-уксусная кислота-пропионовая кислота при атмосферном давлении. - Ж. физ. химии, 1968 , т. 42 , № 5, c. $1104-1108$.

12. Коган В. Б., Фрид ман В. М., Кафаров В. В. Равновесие между жидкостыю и паром. М.-Л., 1966.

13. С а былин И. И., А ри стович В. Ю. Расчет равновесий жидкость-пар многокомпонентных систем. - Ж. прикл. химии, 1970, т. 43, № 9, с. 2021-2027. 
L. KUDRJAVTSEVA, R. TÄHT, O. EISEN

\section{PALJUKOMPONENDILISTE SÜSTEEMIDE AURU - VEDELIKU TASAKAALU ARVUTAMINE}

Artiklis on esitatud meetod, mida saab rakendada paljukomponendiliste süsteemide puhul, mille kaksiksüsteemide suhtelise lenduvuse sõltuvus lahuse koostisest on lähedane lineaarsele. Nimetatud meetodil arvutatud auru koostist ja keemistemperatuuri (aururõhku) on võrreldud katseandmetega ja Wilsoni meetodil arvutatud tulemustega.

L. KUDRJAWZEWA, R. TÄHT, O. EISEN

\section{BERECHNUNG DES GLEICHGEWICHTES VON DAMPF-FLUSSIGKEIT IN MEHRSTOFFSYSTEMEN}

Das im Artikel dargelegte Verfahren eignet sich besonders für die Berechnung der Gleichgewichtsdaten von Mehrstoffsystemen, deren binäre Systeme eine lineare Abhängigkeit der relativen Flüchtigkeit von der Zusammensetzung der Lösung zeigen. Das Verfahren ist am Literaturmaterial geprüft und mit dem Wilson-Verfahren verglichen worden. 